\title{
UN BIOTOPE HYDROBIOLOGIQUE REMARQUABLE : LES LAURONS DE LA CRAU (BOUCHES-DU-RHONE, FRANCE). LA COMMUNAUTE ANIMALE ET SES RELATIONS AVEC LE PEUPLEMENT DES BIOTOPES AQUATIQUES VOISINS
}

\author{
par J. GIUDICELLI' ${ }^{1}$ Z. MOUBAYED ${ }^{2}$ et J. N. TOURENQ ${ }^{2}$
}

La stabilité thermique $\left(12,6^{\circ} \mathrm{C}\right)$ et le renouvellement permanent des eaux des laurons sous un climat méditerranéen permet l'installation d'une faune originale. Par rapport aux communautés palustres voisines on note l'apparition d'espèces sténothermes d'eau froide ayant une répartition boréoalpine et rhéobionte (crénon $\rightarrow$ rhithron $\rightarrow$ potamon) dont les plus typiques sont: Pseudamnicola lanceolata et $P$. anatina (Mollusques), Atractides prosiliens (Hydracariens), Gymnometriocnemus brumalis, Orthocladius rubicundus, Pseudorthocladius curtistylus (Chironomides), Athripsodes dissimilis (Trichoptère).

\section{A remarkable hydrobiological biotope : \\ the natural wells of Crau (mouth of the Rhône, France). The animal community and its relationships with the population of neighbouring aquatic biotopes.}

Thermal stability $\left(12.6^{\circ} \mathrm{C}\right)$ and constant renewal of water in these natural wells under a mediterranean climate has allowed the development of a unique fauna. In comparison with neighbouring swampy communities, there is the presence of cold-water stenotherms that usually have a boreoalpine and rheobiontic distribution (crenon $\rightarrow$ rhithron $\rightarrow$ potamon). The most typical species are : Pseudamnicola lanceolata and $P$. anatina (molluscs), Atractides prosiliens (Hydracarina), Gymnometriocnemus brumalis, Orthocladius rubicundus, Pseudorthocladius curtistylus (chironomids), Athripsodes dissimilis (Trichoptera).

\section{I. - DEFINITION ET ORIGINE DES LAURONS}

Le mot lauron vient du provençal «lauroun » qui signifie surgeon d'eau. Ce sont des puits naturels, ouverts dans les alluvions récentes de la Crau d'où jaillit une eau claire, douce et fraîche qui se répand le plus souvent dans les marais.

Sur la bordure occidentale de la Crau s'étend une zone marécageuse qui est alimentée par de nombreux laurons. Ceux qui ont été étudiés

1. Université d'Aix-Marseille III. Centre de Saint-Jérôme, La boratoire de B.A. Ecologie. 13013 Marseille. France.

2. Laboratoire d'Hydrobiologie, Université P.-Sabatier, 118, route de Narbonne, 31062 Toulouse Cedex. France. 

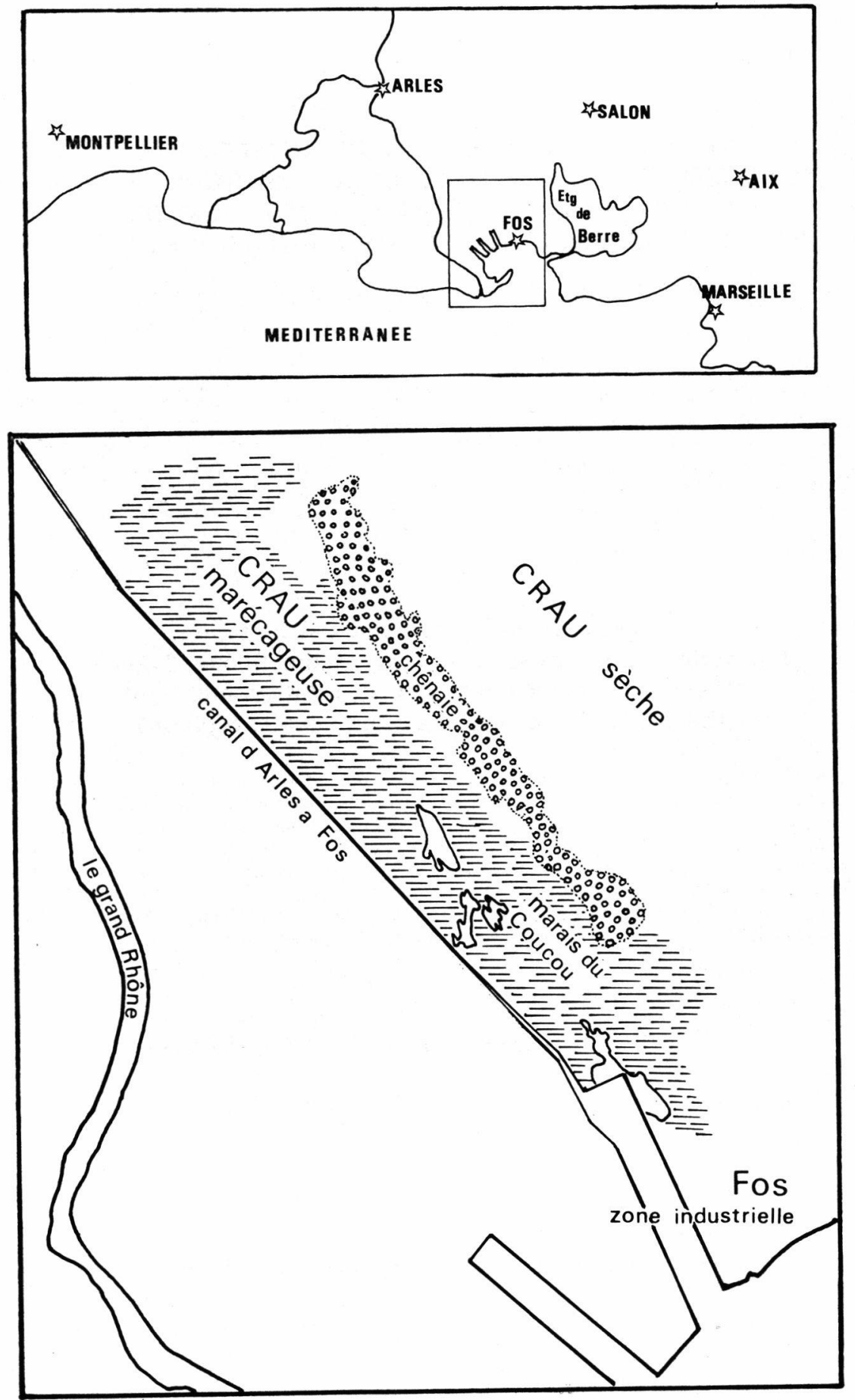

Fig. 1. - Situation géographique des marais du sud de la Crau. 
sont situés dans les marais de la dépression du Viguiérat, au nord du Golfe de Fos (fig. 1).

L'histoire géologique récente et l'hydrologie de la Crau nous montrent l'origine et la formation des laurons et nous expliquent leur fonctionnement.

\subsection{Géologie de la Crau}

La plaine de la Crau est située à l'est du Grand Rhône et forme un triangle dont les pointes sont Arles à l'ouest, Lamanon à l'est et Fos au sud.

Elle est formée par des alluvions grossières apportées par l'ancienne Durance. Jusqu'à la dernière glaciation quaternaire la Durance n'avait pas encore acquis son embouchure actuelle : elle passait entre les massifs des Alpilles et du Lubéron par le seuil de Lamanon, puis se dirigeait vers l'embouchure du Rhône, ver la région de Fos.

Sur un substrat de marnes grises, l'ancienne Durance a déposé des cailloutis d'origine alpine, à la fin du tertiaire (Crau villafranchienne) et au début de la dernière glaciation quaternaire (Crau wurmienne). Ces dépôts, d'une vingtaine de mètres d'épaisseur, sont formés de galets mêlés à des graviers et des sables qui souvent s'agglomèrent en poudingue.

Au niveau des marais de la dépression du Viguiérat, les cailloutis (pente moyenne de 3 pour 1000 , côte $-35 \mathrm{~m}$ ) sont recouverts d'alluvions fluvio-marines de nature sableuse et limoneuse.

\subsection{Hydrologie}

Les marais du Viguiérat sont alimentés par les précipitations, les eaux de surface et les eaux souterraines de la Crau.

Les eaux de surface sont amenées depuis les Alpilles par un réseau de canaux d'irrigation; elles empruntent aussi le canal de navigation d'Arles à Fos et le canal de drainage du Viguiérat.

Les eaux souterraines comprennent:

- la nappe phréatique superficielle, située dans les sables et les limons de la zone des marais,

- la nappe profonde, ou nappe de Crau, située dans la couche de cailloutis déposée par l'ancienne Durance.

Leś eaux de la nappe de Crau s'écoulent suivant une direction nordest - sud-ouest, depuis le seuil de Lamanon vers la zone des marais de Fos; elles circulent et se mettent en charge dans les sables et graviers du cailloutis. Lorsqu'elles peuvent trouver un exutoire à travers la 
carapace imperméable formée par le cailloutis (fissures, discontinuités entre les lentilles du poudingue), elles montent "per ascensum » dans ia zone des marais. Ces eaux souterraines sous pression traversent alors la couche limono-sableuse et, par des puits verticaux en forme de tronc de cône, constituent les laurons (fig. 2) qui contribuent, en toutes saisons, à l'alimentation des marais.

En saison des pluies s'ajoute l'apport de la nappe phréatique superficielle.

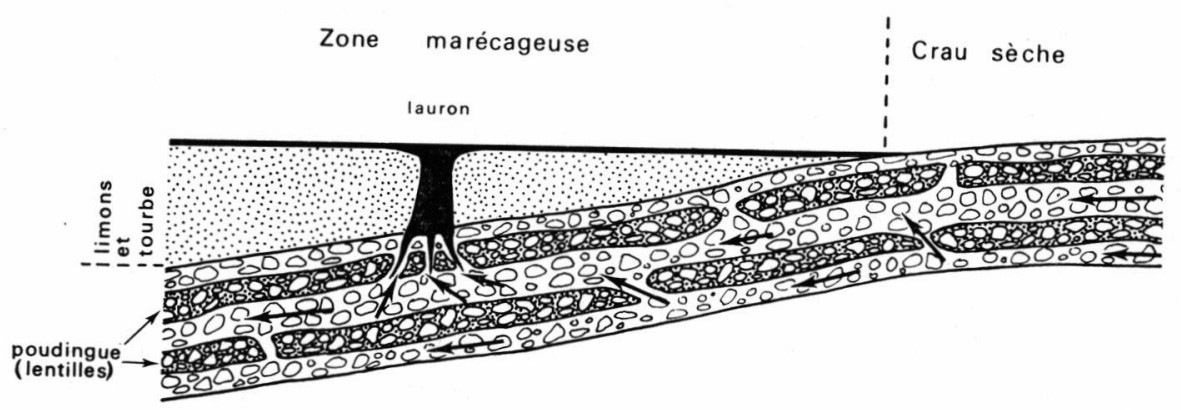

I'ig. 2. - Coupe schématique d'un lauron (les flèches indiquent la circulation de l'eau de la nappe profonde dans les cailloutis).

\section{2. - CARACTERISTIQUES MORPHOLOGIQUES DES LAURONS}

Les laurons étudiés sont situés dans les marais du Coucou (fig 3), au sein d'un groupement végétal hydromorphe à Cladium mariscus, Schoenus nigricans, Juncus striatus (Metge 1977). Leurs puits, creusés dans les tourbes et les limons, ont un diamètre externe compris entre 50 et $200 \mathrm{~cm}$, une profondeur comprise entre 150 et $230 \mathrm{~cm}$.

Deux types de laurons, d'après leurs caractéristiques hydrologiques, physiographiques, floristiques, sont définis.

\subsection{Les laurons ouverts}

Ce sont des laurons typiques. Leur régime hydrique est normal, avec un débit élevé pouvant atteindre, pour les plus importantes, $1 \mathrm{~m}^{3} / \mathrm{sec}$. Le fond est clair, constitué de sables et de graviers provenant de l'érosion des éléments du poudingue et des cailloutis par la pression de l'eau lors des grands débits de printemps. La hauteur de la lame d'eau est comprise entre 200 et $250 \mathrm{~cm}$.

La végétation est localisée sur le bord externe du puits, où poussent 
Cladium mariscus (hélophyte) et Potamogeton lucens (hydrophyte). Le fond et les parois du puits sont nus, totalement dépourvus de végétaux.

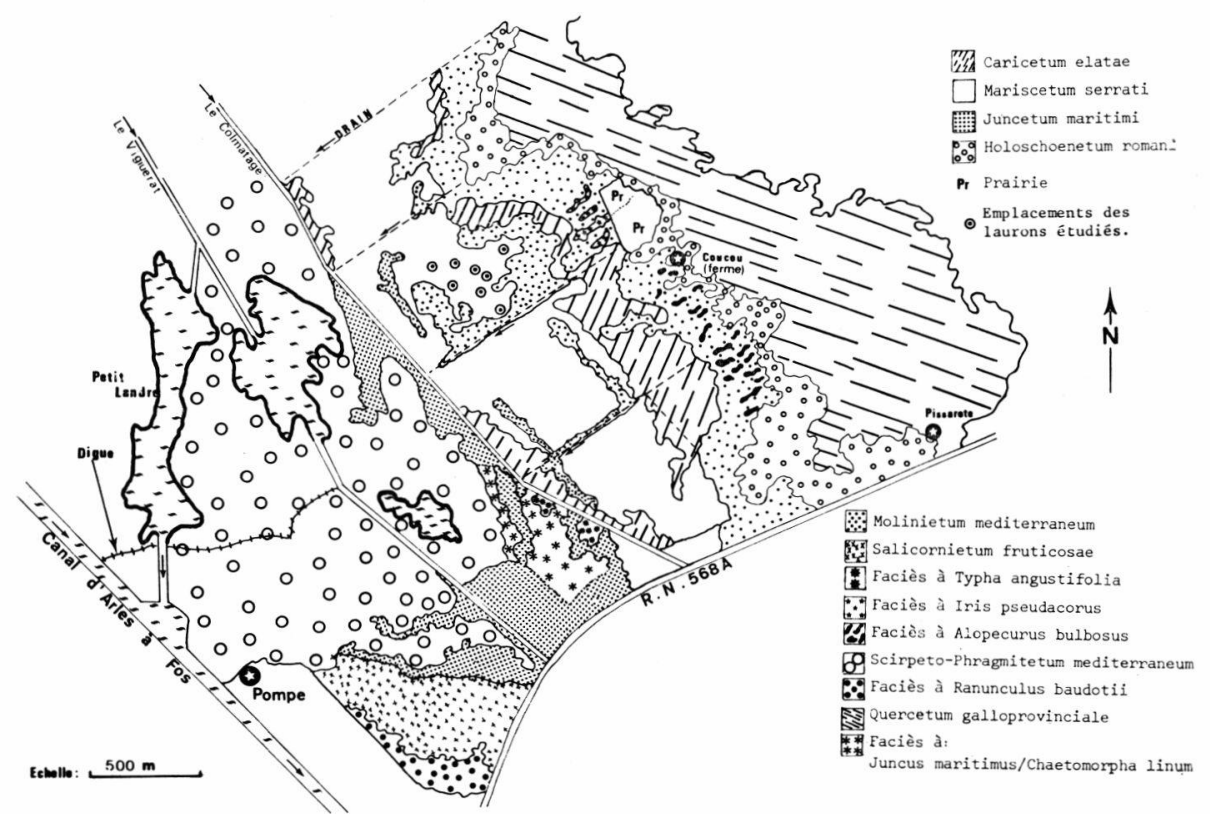

Fic. 3. - Carte phytoécologique des marais du Coucou avec emplacement des laurons.

\subsection{Les laurons colmatés}

Le débit est faible et le renouvellement de l'eau dans le puits est trop lent pour s'opposer au dépôt d'éléments allotchones provenant des marais : végétaux morts et débris résultant du faucardage de la végétation palustre environnante.

Le fond, limono-humo-sableux avec une faible fraction de sable, est de couleur brune. Ce substrat permet l'enracinement et l'installation de Nymphaea alba dont la nappe foliaire parvient, au printemps, à recouvrir presque entièrement la surface du lauron.

La hauteur d'eau varie entre 130 et $160 \mathrm{~cm}$.

Dans les deux types de laurons vit une flore algale de diatomées, d'algues vertes filamenteuses et d'algues brunes. La densité de cette flore est plus grande dans les laurons ouverts qui reçoivent plus de rayonnement solaire (absence de végétaux aquatiques de surface). Sur les tiges immergées de Cladium, sur les tiges et les feuilles de Nymphaea est fixé toute l'année un important périphyton. 


\section{3. - COMPOSANTES THERMIQUES ET HYDROCHIMIQUES}

Les eaux des laurons diffèrent des eaux des marais environnants par:

\subsection{La température}

Elle a été mesurée à $20 \mathrm{~cm}$ au-dessous de la surface.

Les eaux des laurons sont remarquables par leur constance thermique dans le cycle journalier et dans le cycle annuel.

La moyenne annuelle s'établit à $12^{\circ} 6$, le maximum thermique à $13^{\circ}$ en juin et le minimum à $11^{\circ} 9$ en décembre.

En été, les eaux des laurons sont plus fraîches que celles de leur lisière, l'écart thermique entre les deux biotopes atteignant $10^{\circ}$ en juillet 1977 ; en certaines stations du marais la température de l'eau s'élève jusqu'à $33^{\circ}$ en juillet.

Le profil thermique des marais du Coucou(fig. 4), établi sur un

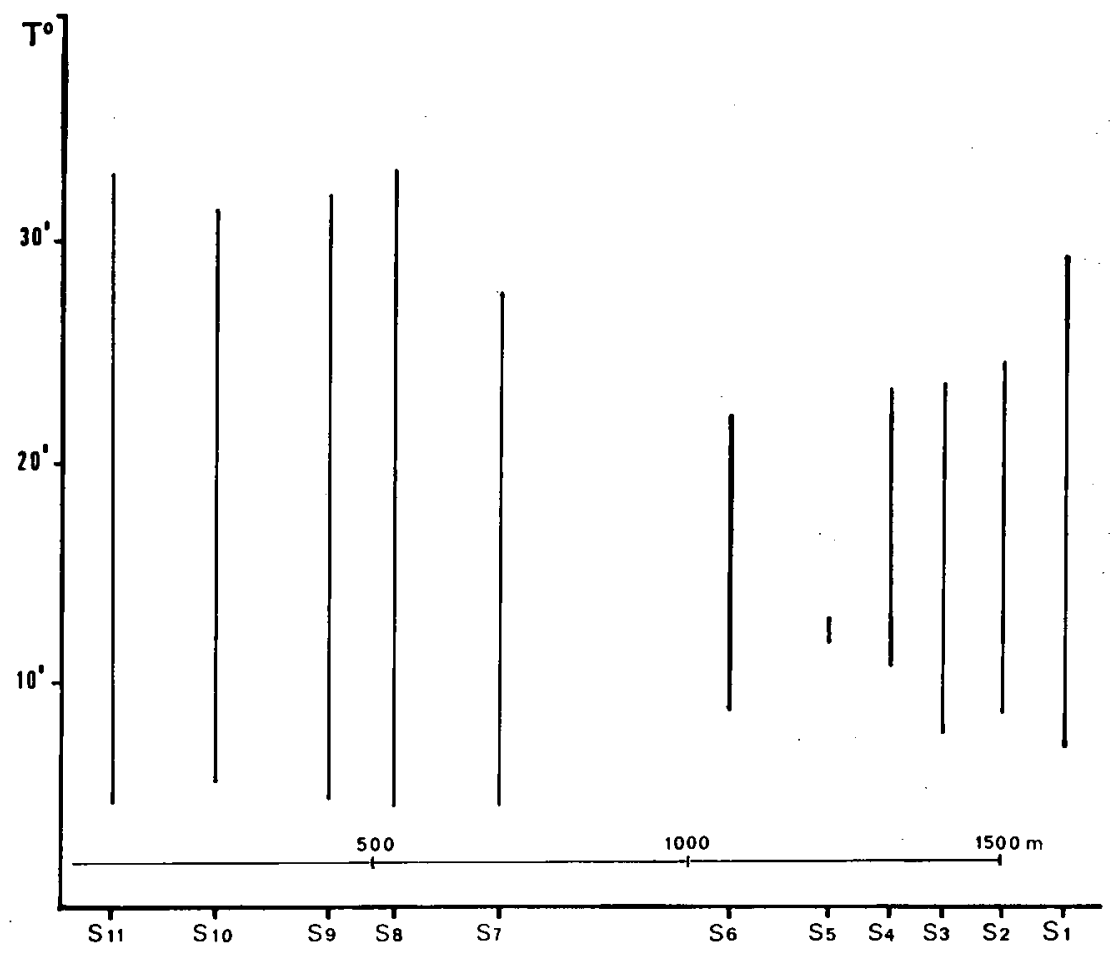

Fig. 4. - Profil thermique des marais du Coucou (11 stations sur un transect estouest (la station S5 correspond aux laurons ouverts, la station S6 aux laurons colmatés). 
transect passant par 11 stations, montre l'originalité et la stabilité thermique des eaux des laurons ouverts (station $\mathrm{S} 5$ ). Le régime thermique de ces eaux d'origine souterraine influence celui des biotopes aquatiques permanents et semi-permanents voisins (stations $\mathrm{S} 4$ et S 2 , S 3) où les écarts de température sont réduits et les minima de l'ordre de $10^{\circ}$. En revanche, dans les stations plus éloignées des laurons (stations S 1, S 7 à S 11), la température des eaux dépend de celle de l'atmosphère ; les différences entre les minima et les maxima thermiques sont importantes.

En hiver, les eaux sont plus chaudes que celles du marais environnant; des vapeurs se forment alors au-dessus des laurons de novembre à janvier.

Dans les laurons colmatés (station S6), l'amplitude thermique annuelle est plus grande (minimum : $9^{\circ}$, maximum: $21^{\circ} 2$ ), mais les eaux restent relativement fraîches en été.

\subsection{L'oxygène dissous}

La teneur en oxygène dissous est dans les laurons toujours voisine de la saturation. Elle reste constante tout au long de l'année avec des valeurs comprises entre 82 et $98 \%$ de saturation. Les teneurs en oxygène dissous mesurées dans les marais du Coucou, au voisinage des laurons, montrent des variations tantôt faibles (entre 30 et $60 \%$ de saturation), tantôt forte (entre 25 et $190 \%$ ), notamment dans les biotopes à Cladium mariscus et à Carex elata.

\subsection{Les sels dissous}

Les graphiques ( $f$ ig. 5 et 6 ) représentent les teneurs des différents ions et leurs évolutions sur un cycle annuel des eaux des laurons ouverts et des laurons colmatés.

On note la richesse des eaux en calcium et les variations des chlorures en cours d'année (influence du lessivage des sols pendant la saison des pluies et proximité du biseau salé à la partie méridionale du marais qui se déplace selon les besoins des pompages industriels).

Les variations chimiques des eaux des marais voisins sont représentées sur les graphiques (fig. 7).

Le tableau ( $f i g$ 8) résume l'évolution de la composition chimique des eaux des laurons. En août la composition chimique des eaux des laurons se rapproche bien de celle de la nappe de la Crau. Au printemps et en automne ce sont surtout les influences de la nappe superficielle (lessivage par les pluies) qui provoquent le décalage et la rapprochent alors de celle des eaux des marais de la station 10. 


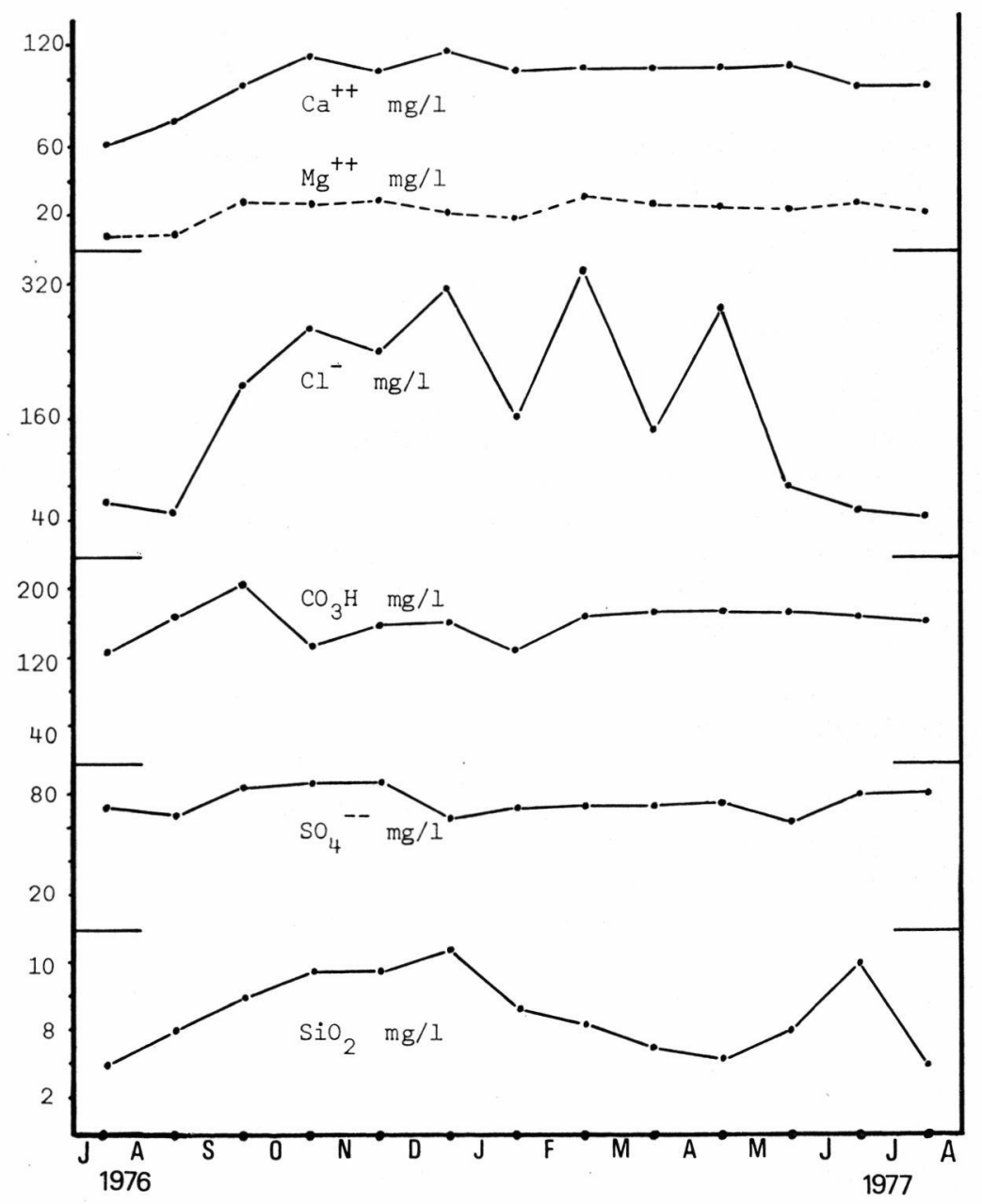

Fig. 5. - Evolution des caractères chimiques des eaux des laurons ouverts (S5). 


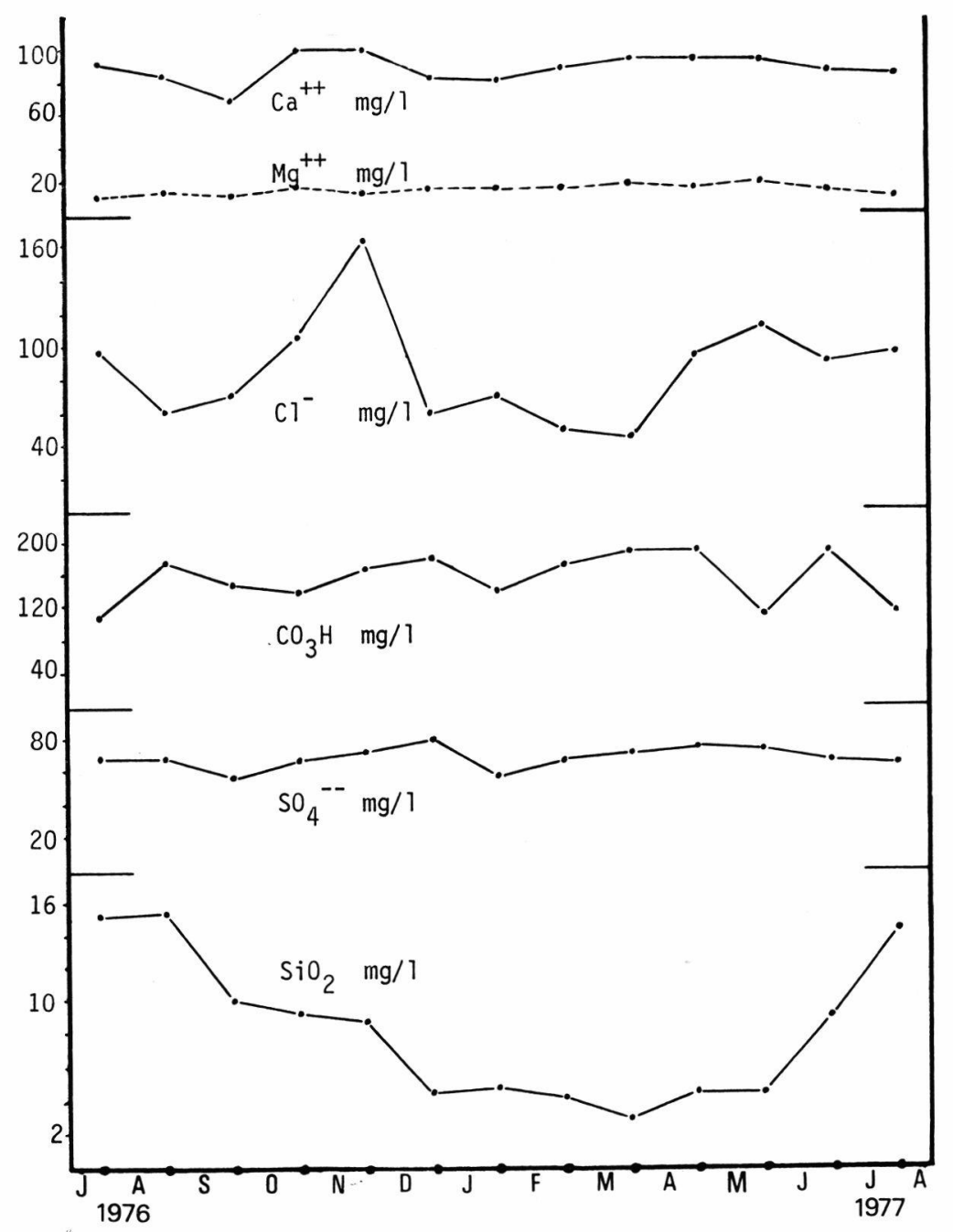

Fig. 6. - Evolution des caractères chimiques des eaux des laurons colmatés (S6). 


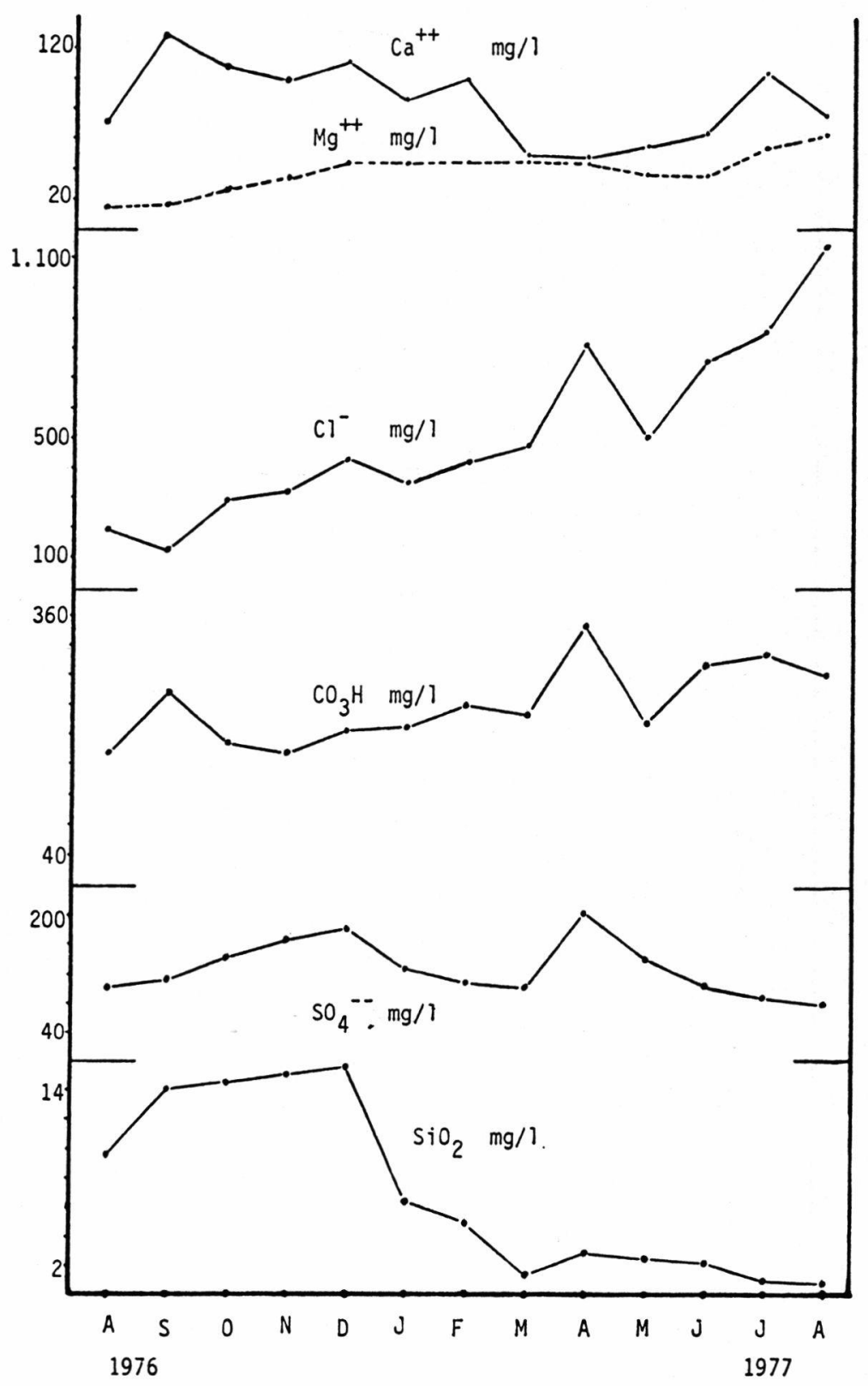

Fig. 7. - Evolution des caractères chimiques des eaux des marais proches des laurons (S8, S9, S10, S11). 


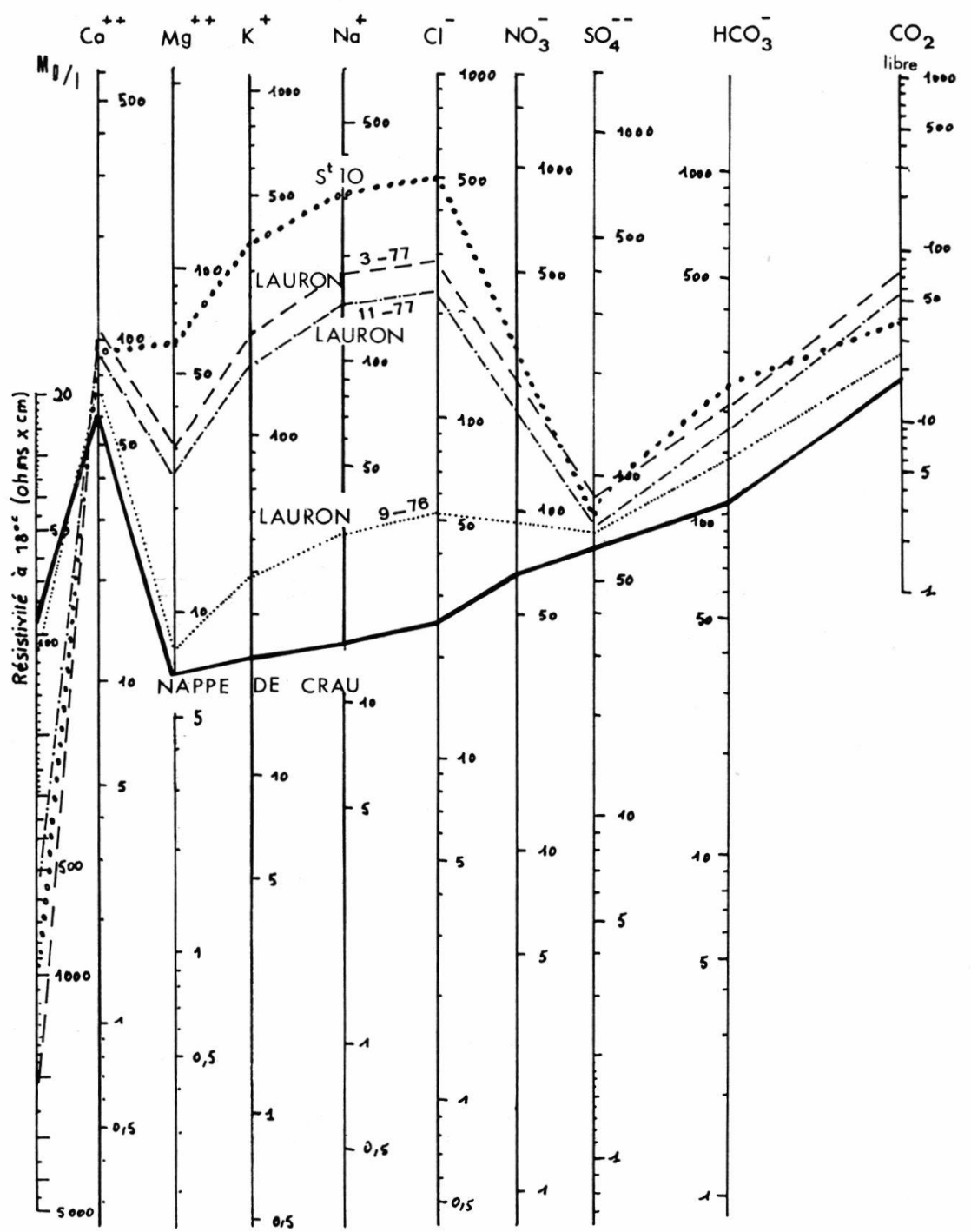

Fig. 8. - Evolution en cours d'année de la composition chimique des eaux des laurons (comparaison avec la composition chimique des eaux de la nappe de Crau et d'un marais voisin des laurons (S10).

\section{4. - LA COMMUNAUTE ANIMALE DES LAURONS}

Les botanistes, en particulier Molinier et Tallon (1949), ont relevé dans ces marais du sud de la Crau la présence d'espèces boréoalpines.

Ce sont : 
- Dryopteris phegopteris (Fougère)

- Parnassia palustris (Saxifragacée)

- Gentiana pneumonanthe (Gentianacée).

Iis ont émis l'hypothèse que ces espèces représentent des reliques glaciaires qui se seraient maintenues dans les marais grâce à la présence des eaux froides des laurons.

Il était intéressant de rechercher, dans le peuplement animal, d'éventuelles originalités faunistiques et, en particulier, l'existence de formes sténothermes d'eau froide qui pourraient être les homologues des végétaux boréoalpins cités plus haut.

Nous essaierons de faire apparaître l'originalité de ce peuplement en le comparant à celui du marais.

Les prélèvements de faune ont été effectués d'août 1976 à août 1977, à raison d'un relevé tous les 20 jours environ. Dans le marais la faune benthique et la faune nageuse ont été prélevées à l'aide d'un cylindre creux de $25 \mathrm{~cm}$ de diamètre, isolant une surface de substrat et un volume d'eau connus. Dans les laurons l'échantillonnage a été réalisé au moyen d'un filet troubleau.

\subsection{Structure globale de la communauté et comparaison avec les communautés palustres}

Dans les laurons, comme dans les autres biotopes aquatiques des marais du Coucou, trois groupes dominent en effectif : les Crustacés, les Mollusques (essentiellement les Gastéropodes), les Diptères (essentiellement les Chironomides).

249 espèces ou unités systématiques ont été identifiées dans les laurons et leurs abords. Leur liste complète est récapitulée dans le travail de Moubayed (1978) : Plathelminthes (4), Mollusques (29), Annelides (20), Arachnides (20), Crustacés (19), Ephéméroptères (2), Odonates (12), Hétéroptères (19), Coléoptères (54), Trichoptères (9), Lépidoptères (6), Chironomides (54), Diptères divers (6).

Les relations quantitatives entre les différents groupes zoologiques dans les laurons ouverts et colmatés, d'une part, et dans 5 autres stations franchement palustres, d'autre part, sont représentés sous la forme de spectres d'abondance (fig. 9).

Les stations soumises à comparaison sont les suivantes :

- $\mathrm{S}_{1}$ : station périphérique, biotope à submersion temporaire (à sec de mai à septembre) ; faciès à Alopecurus bulbosus et Juncus articulatus.

- $\mathrm{S}_{3}$ : biotope à submersion semi-permanente (submersion de 10 mois, le sol reste imbibé d'eau pendant l'été); faciès à Carex elata et Schoenus nigricans. 

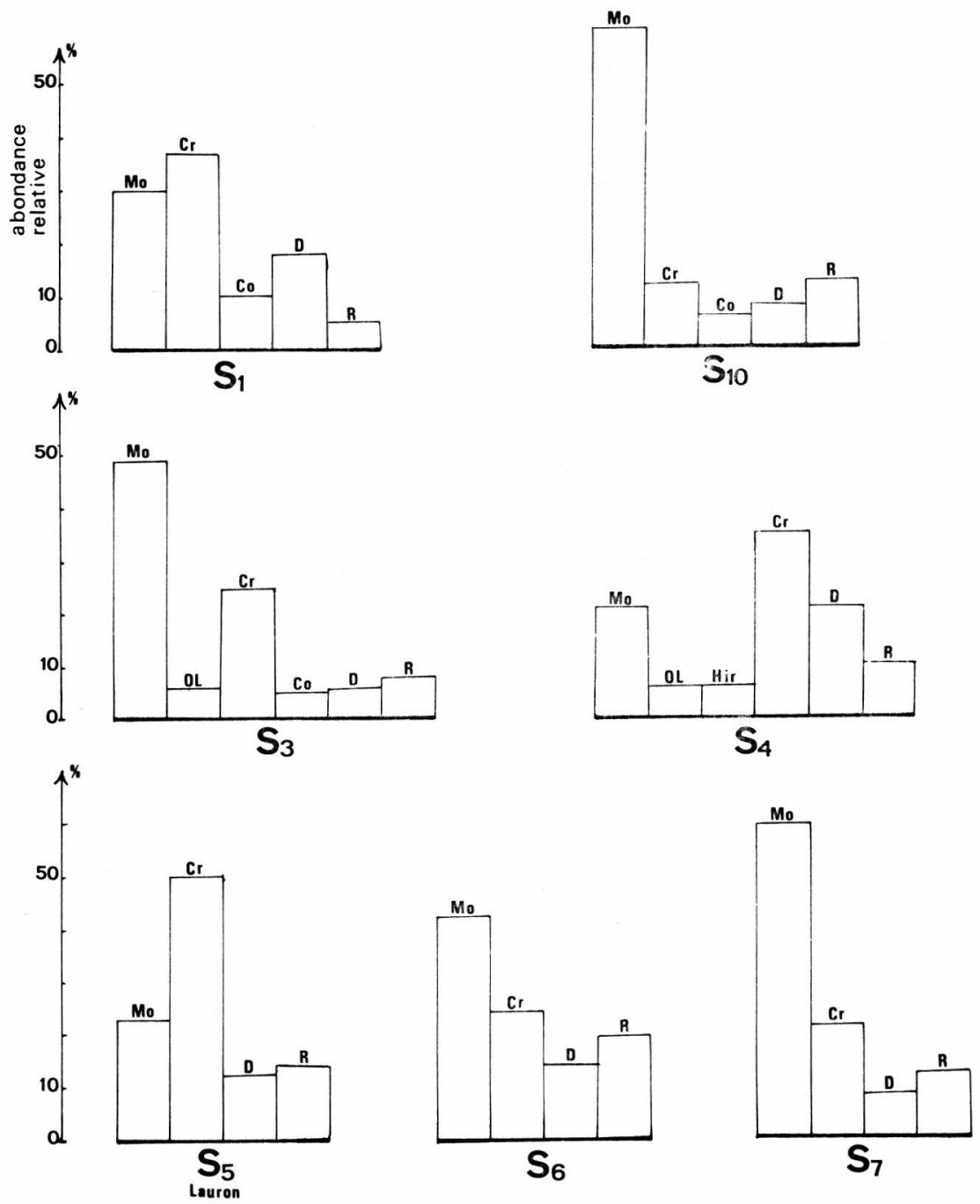

Fig. 9. - Structure des communautés stationnelles en fonction de l'abondance relative des principaux groupes zoologiques. Mo: Mollusques; $\mathrm{Cr}$ : Crustacés; $\mathrm{Ol}$ : Oligochètes ; Hir : Hirudinées ; Co : Coléoptères ; D : Diptères ; R : autres groupes zoologiques.

- $\mathrm{S}_{4}$ : biotope d'eau permanente; faciès à Carex elata et Cladium mariscus.

- $\mathrm{S}_{\overline{5}}$ : lauron ouvert - une dizaine de laurons se trouvent réunis dans cette station; les prélèvements ont eu lieu dans trois d'entre eux.

$-S_{6}$ : lauron colmaté. 
$-\mathrm{S}_{7}$ : biotope d'eau permanente; faciès à Carex elata, Nymphea alba, Chara.

$-S_{10}$ : station périphérique, biotope à submersion semi-permanente ; faciès à Chara et Juncus maritimus.

La dominance des Crustacés caractérise le peuplement des laurons ; les Mollusques viennent au second rang avec, cependant, une abondance relative plus faible que dans la plupart des autres stations du marais où les représentants de ce groupe constituent la moitié, ou plus, de l'effectif des individus prélevés sur un cycle annuel. Ainsi, sur un cycle annuel, 25220 individus répartis en 29 espèces, figurent dans nos prélèvements. Les effectifs les plus élevés s'observent dans les biotopes à submersion semi-permanente (5990 individus à $\mathrm{S}_{3}$ ). Les biotopes où la hauteur d'eau est importante hébergent les populations malacologiques les plus réduites (470 individus à $\mathrm{S}_{5}, 695$ à $\mathrm{S}_{6}$ ), bien que la diversité spécifique soit aussi élevée qu'à la station $S_{3}$.

Pour ces deux groupes la dominance est le fait de quelques espèces:

- à $\mathrm{S}_{1}$ : Anisus rotundatus (Gast.), Candona neglecta (Crust. Ostracodes).

- à $\mathrm{S}_{3}$ : Anisus rotundatus, Pseudamnicola compacta (Gast.), Sphaerium lacustre (Lamellibranche), Eucypris virens (Crust. Ostracodes), Simocephalus vetulus (Crust. Cladocères).

- à $S_{4}$ : Eucypris virens, Simocephalus vetulus.

- à $S_{5}$ : (laurons ouverts): Pseudamnicola anatina, $P$. lanceolata (Gast.), Alona affinis, Eurycercus lamellatus (Crust. Cladocères).

- à $\mathrm{S}_{\mathbf{6}}$ : (laurons colmatés) : Bithynia tentaculata, Pseudamnicola anatina (Gast.), Pisidium casertanum (Lamellibr.).

- à $S_{7}$ : Bithynia tentaculata, Pseudamnicola anatina, Valvata cristata, Pisidium casertanum (Mollusques).

- à $S_{10}$ : Pseudamnicola compacta, Bithynia tentaculata, Lymnaea ovata, Physa acuta, Planorbis planorbis (Gast.).

En fait, dans les laurons ouverts, les espèces dominantes sont des formes nageuses, comme les Cladocères planctoniques Alona et Eurycercus. Dans les autres stations du marais, où la lame d'eau est peu épaisse, dominent des espèces benthiques; ce sont pour la plupart des Gastéropodes (Anisus, Pseudamnicola, ...), des Lamellibranches fouisseurs (Pisidium, Sphaerium) et aussi des Ostracodes comme Candona neglecta qui, à la station $\mathrm{S}_{1}$, ont été récoltés dans les prélèvements de substrat superficiel.

Les Coléoptères, dans les laurons et leurs abords $\left(\mathrm{St}_{\mathrm{j}} \mathrm{S}_{5}, \mathrm{~S}_{6}, \mathrm{~S}_{7}\right)$, ont une représentation très modeste et une faible diversité spécifique, comparativement au peuplement des biotopes palustres semi-permanents et temporaires. Ainsi, pour l'ensemble des prélèvements de 1976 on relève : 26 individus et 5 espèces à $S_{\overline{5}}, 31$ individus et 7 espèces à 
$\mathrm{S}_{6}$ contre 702 individus et 25 espèces à $S_{1}, 586$ individus et 35 espèces à $\mathrm{S}_{3}$.

Les biotopes palustres peu profonds, plus ou moins intermittents, sont donc les plus peuplés en Coléoptères. Les facteurs trophiques expliquent probablement cette richesse et cette diversité. Ainsi, les Haliplides, dont les larves sont végétariennes, sont cantonnés dans les stations riches en Chaetomorpha et en Chara $\left(S_{3}\right.$ et $\left.S_{10}\right)$. Les Dytiscides, qui sont des carnassiers, dominent dans les stations $\mathbf{S}_{1}$ et $\mathbf{S}_{3}$ où ils trouvent une nourriture abondante de petits Crustacés et de iarves de Chironomides. Les Coléoptères de ces biotopes temporaires et semi-temporaires sont d'ailleurs des formes migratrices qui arrivent en masse au printemps dans ces stations lorsque les ressources alimentaires sont les plus abondantes.

Les biotopes permanents, où la hauteur d'eau est maximum (laurons et leurs abords), offrent une nourriture animale plus réduite:

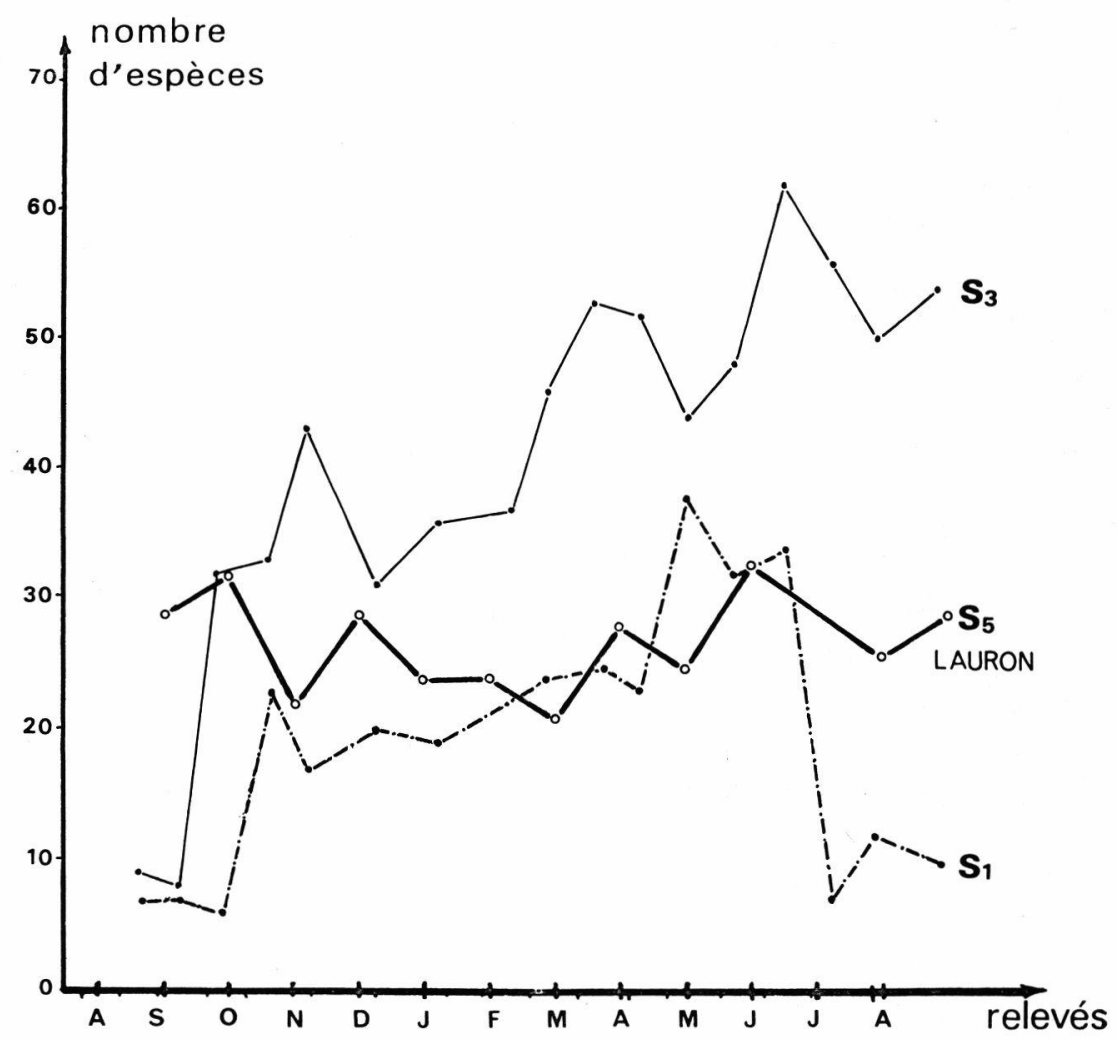

Fig. 10. - Evolution de la richesse en espèces par relevé dans les stations S1, S3 et S5. 
1000 individus de Crustacés et 247 larves de Chironomides récoltés en 1976 à $S_{5}$, contre 2598 et 1256 à la station $S_{1}$.

Une autre particularité de la communauté des laurons est la présence de nombreuses espèces de Trichoptères. Ce groupe réalise sa densité et sa diversité maximum dans ce biotope qui héberge 7 espèces et 78 individus sur les 9 espèces et les 150 individus qui ont été recensés dans l'ensemble des stations des marais du Coucou. Dans les biotopes palustres permanents et temporaires de ce secteur, les Trichoptères sont représentés par 3 espèces d'eau stagnante des genres Limnephilus et Phryganea; dans les laurons on ne trouve que des Trichoptères Hydroptilides et Leptocérides qui vivent généralement dans ies cours d'eau lents.

L'évolution du nombre d'espèces par relevé dans le cycle annuel (fig. 10) montre que dans les laurons la richesse en espèces varie assez peu; ceci traduit une relative stabilité du peuplement. Dans les biotopes temporaires $\left(S_{1}\right)$ il se produit d'importantes fluctuations de la richesse, en particulier une diminution brutale au moment de l'assèchement estival. De fortes fluctuations, accompagnées d'un évolution ascendante de la richesse, s'observent dans les biotopes semi-permanents $\left(\mathbf{S}_{3}\right)$.

Les courbes de richesse cumulée, obtenues en dénombrant et en cumulant les espèces nouvellement apparues dans les prélèvements successifs d'un cycle annuel, permettent de suivre et de comparer l'évolution et la structure des différentes communautés des marais du Coucou (fig. 11).

Les communautés des biotopes palustres permanents $\left(S_{10}\right)$, semipermanents $\left(S_{3}\right)$ et temporaires $\left(S_{1}\right)$ sont caractérisées par un accroissement rapide de la richesse et un taux de changement faunistique élevé pendant les 4 premiers mois du cycle hydrologique, le palier étant atteint en avril-mai. Au début du cycle, les communautés sont pauvres en espèces; le premier prélèvement renferme entre 7 et 18 espèces soit, pour certaines communautés, le quart et, pour d'autres, le dixième de la richesse totale.

Dans les laurons ouverts ou colmatés les courbes débutent avec un effectif élevé ( 28 et 34 espèces au premier prélèvement) qui représente à peu près la moitié de la richesse totale du peuplement.

\subsection{Les espèces caractéristiques des laurons et leur signification écologique}

La comparaison des peuplements des différents biotopes inventoriés dans les marais du Coucou (13 stations) nous a permis de reconnaître, au sein des principaux groupes d'Invertébrés, plusieurs espèces carac- 


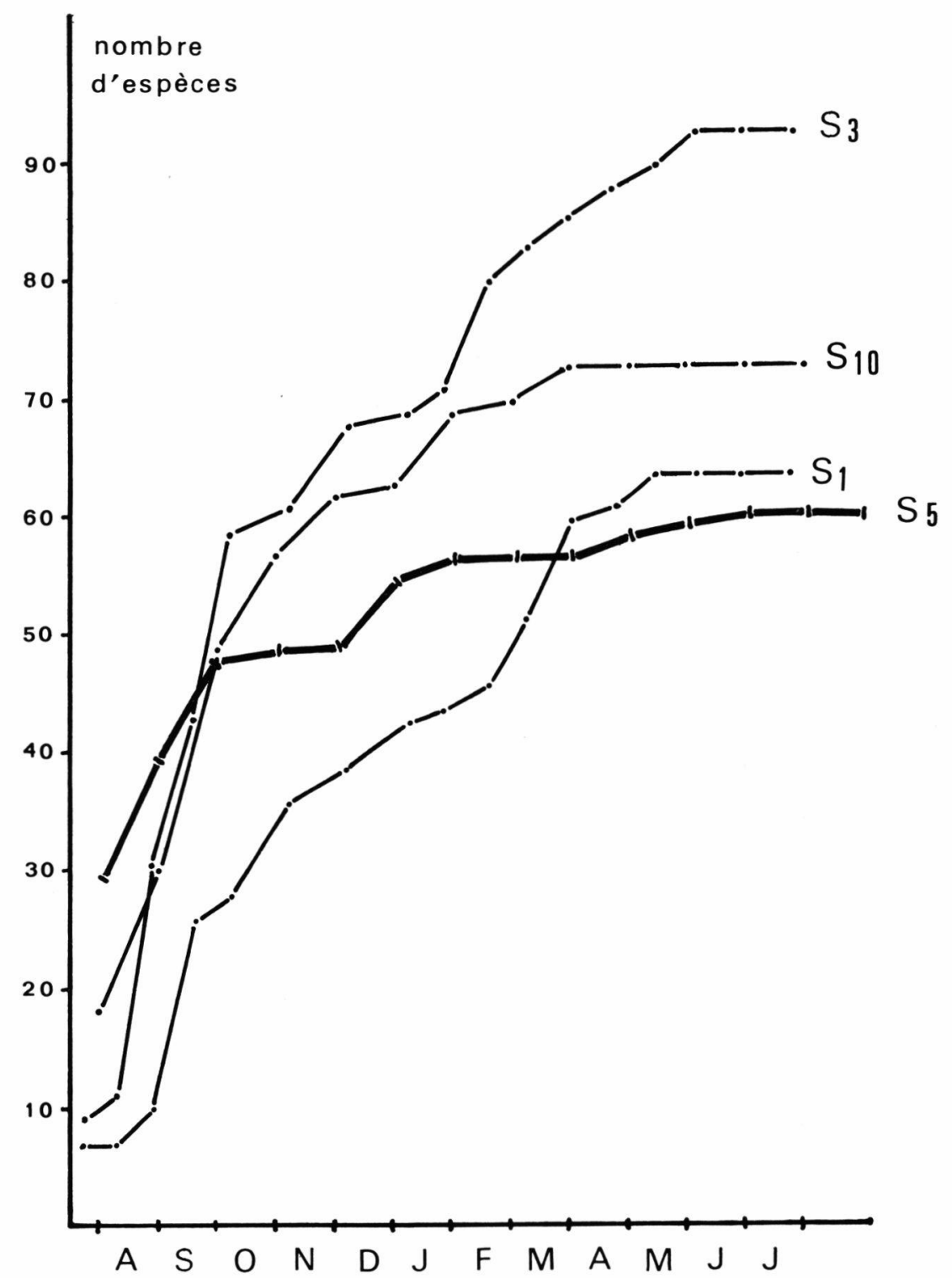

Fig. 11. - Représentation graphique de la richesse cumulée dans un cycle hyarologique pour les stations S1, S3, S5 et S10.

téristiques des laurons. Certaines sont strictement localisées dans ce biotope; d'autres sont présentes ailleurs dans le marais, mais elles réalisent leur densité maximale dans les laurons et y trouvent leur optimum écologique. 
En se référant à nos connaissances sur l'autoécologie de ces espèces nous verrons, pour certaines d'entre elles, dans quelle mesure leu présence traduit les particularités mésologiques du biotope des laurons. Ceci nous conduira à mieux préciser, d'une part, l'originalité de son peuplement et, d'autre part, sa signification écologique et biogéographique dans le domaine limnique méditerranéen.

Les espèces caractéristiques ou indicatrices apparaissent dans la plupart des groupes zoologiques, les Chironomides fournissant le lot le plus important.

\section{OLIGOCHÈTES}

Sur les 12 espèces recensées dans la zone d'étude, trois sont exclusivement cantonnées dans les laurons $\left(\mathrm{S}_{5}\right.$ et $\left.\mathrm{S}_{i}\right)$.

Potamothrix bavaricus, selon Timm (1970), vit dans la vase des eaux douces et saumâtres et, selon Giani (in litt.), semble bien supporter une pollution organique.

Potamothrix heuscheri vit en rivière dans le sable et la vase (Timm, ibid.) et aussi dans les zones eutrophes (Lang 1975).

Slavina appendiculata est une espèce plus intéressante, puisque, selon Cekanoskaya (1965: in Särkä 1978) elle est typique des tourbières à sphaignes; elle serait donc inféodée à des eaux plus froides.

\section{Mollusoues}

Sur les 23 espèces récoltées dans le secteur du Coucou, 5 n'ont pas été signalées en Camargue par Marazanof (1969) ; parmi celles-ci, quatre sont localisées dans les laurons et leurs alentours : Viviparus lacustris, Planorbis carinatus, Pseudamnicola lanceolata, Physa fontinalis.

En fait, les laurons hébergent des espèces inféodées aux eaux claires et pures, stagnantes ou à faible courant, relativement fraîches. On retiendra surtout les espèces qui réalisent leur optimum écologique dans ce biotope, certaines étant présentes aussi dans les stations les plus proches $\left(\mathbf{S}_{4}\right.$ et $\left.\mathbf{S}_{7}\right)$ :

Planorbis carinatus, Anisus albus, A. complanatus, Viviparus lacustris, Physa fontinalis, Pseudamnicola anatina, P. lanceolata, Pisidium casertanum.

La présence des deux espèces de Pseudamnicola est écologiquement significative puisqu'elles ont pour habitat normal les sources et les ruisseaux (Willmann et Pieper 1978).

On remarque un remplacement d'espèces, au sein d'un même genre, entre le marais et le secteur des laurons. Ainsi, Physa acuta, qui forme d'importantes populations dans les biotopes palustres semi-permanents $\left(S_{2}, S_{9}, S_{10}\right)$, est remplacée par $P$. fontinalis au niveau des laurons $\left(\mathrm{S}_{4}, \mathrm{~S}_{5}, \mathrm{~S}_{6}, \mathrm{~S}_{7}\right)$; de même, Pseudamnicola compacta et $P$. similis sont remplacées par $P$. anatina et $P$. lanceolata, Anisus crista et $A$. 
contortus par $A$. albus et $A$. complanatus, Pisidium personatum par $P$. casertanum.

\section{Crustacés}

L'inventaire a révélé 18 espèces, à l'exception des Copépodes qui n'ont pas été déterminés. Deux espèces n'ont été récoltées que dars les laurons ouverts :

- Alona affinis (Cladocère) développe une importante population à la station $S_{5}$. Bien que cosmopolite et assez eurytope, cette espèce n'est pas rare dans les eaux froides et renouvelées (Pacaud 1939); Rey (1969) la qualifie même d'hémisténotherme. Ce petit Crustacé nageur trouve dans les laurons ouverts une nourriture appropriée : diatomées et débris organiques provenant de la décomposition des agues filamenteuses et mis en suspension par le renouvellement continu de l'eau.

- Atyaephyra desmarestii (Décapode) : cette petite crevette se trouve fréquemment, en Provence, dans le potamon, y compris dans le Rhône. Dans les laurons, la profondeur de l'eau et son renouvellement sont des facteurs favorables à l'espèce.

On doit signaler l'absence du Cladocère Simocephalus vetulus dans les laurons et leurs abords. Cette espèce eurytherme, capable de supporter d'importantes variations de la teneur en oxygène dissous, abondante dans tous les biotopes palustres du Coucou, est exclue de la zone des laurons par la température de l'eau.

La rareté des Ostracodes dans ce biotope (une seule espèces présente: Eucypris virens) est, elle aussi, remarquable, alors que ce groupe se manifeste, dans les différentes stations palustres du Coucou, par une abondance et une diversité spécifique élevées. Les collections d'eau permanente et profonde semblent peu convenir à ces petits Crustacés.

\section{HYDRACARIENS}

Les 16 espèces récoltées dans la zone des marais du Coucou sont, pour la plupart, des formes d'eau stagnante.

Trois espèces sont exclusives des laurons et caractérisent bien ce biotope: toutes sont des formes sténothermes. Elles apportent d'autres informations écologiques:

- Atractides prosiliens est un hydracarien phréaticole (Viets 1978) et sa présence ici traduit bien l'origine souterraine de l'eau.

- Midea orbiculata vit dans les lacs et aussi dans le rhithron (Viets, ibid.).

- Oxus oblongus, seul représentant du genre dans le secteur du Coucou, trouve dans les laurons son habitat d'élection; en effet, les 
représentants du genre sont qualifiés d' " excellents nageurs " par Motas (1929).

\section{ODONATES}

Les prélèvements de faune benthique et les chasses d'imagos nous ont apporté 12 espèces pour l'ensemble de la zone du Coucou. Toutes ont été signalées en Camargue par Aguesse $(1961,1968)$ à l'exception de Ceriagrion tenellum que nous avons trouvée dans les deux types de laurons et dans le "canal du colmatage » (les adultes ont toujours été capturés aux alentours de ces biotopes).

Aguesse (1968) attribue comme habitat à cette espèce les eaux stagnantes et les ruisseaux à courant lent; il ajoute que sa répartition écologique mériterait d'être précisée. Bigot (com. verb.) a capturé des adultes autour de grosses résurgences fraîches $\left(15^{\circ} \mathrm{C}\right)$ en haute Crau (région de Mouriès). Ces observations et nos récoltes montrent que cet Odonate a des affinités pour les eaux à écoulement lent et plutôt fraîches.

\section{LÉPIDOPTÈRES}

Les larves aquatiques de Nymphula nymphaeata sont caractéristiques des laurons colmatés. Leur présence semble liée au développement des peuplements de nénuphars (Nymphaea alba) dont les feuilles, dans ce biotope, couvrent environ $80 \%$ de la surface du plan a'eau au printemps.

\section{T'RICHOPTĖRES}

Dans les marais du Coucou, les 9 espèces inventoriées se répartissent en 3 familles.

Une espèce nouvelle pour la science, Hydroptila giudicellorum Botosaneanu, 1980 a été découverte dans ce biotope ; il s'agit d'une forme proche de $H$. tineodides, espèce d'Europe occidentale, septentrionale et centrale.

Les Limnephides sont typiquement, à l'état préimaginal, des organismes d'eau calme; leur présence est normale dans les biotopes palustres temporaires et permanents (stations $\mathrm{S}_{1}, \mathrm{~S}_{4}, \mathrm{~S}_{6}, \mathrm{~S}_{7}, \mathrm{~S}_{10}$ ).

Les Hydroptilides et les Leptocérides, cantonnés dans les laurons et leurs abords, traduisent deux types d'influences:

- Les Hydroptilides (Hydroptila giudicellorum, Oxyethira flavicornis, Tricholeiochiton fagesi, Agraylea sexmaculata) sont habituellement des organismes vivant dans les biotopes lotiques. Le renouvellement et l'écoulement de l'eau, la température relativement basse déterminent leur présence dans les laurons. Ils y trouvent, en outre, un périphyton abondant et surtout des algues filamenteuses dont 
les contenus cellulaires sont consommés par les larves (Nielsen 1942, Giudicelli et Vaillant 1967) et dont les filaments servent à la construction des étuis larvaires d'Agraylea. $H$. giudicellorum, l'espèce la plus abondante, trouve dans le substrat des laurons le sable fin indispensable à la construction des étuis.

- Les Leptocérides (Athripsodes dissimilis et Ceraclea senilis) n'ont été récoltés, à l'état larvaire, que dans la station $\mathrm{S}_{5}$. A. dissimilis est caractéristique du cours inférieur des rivières (épi- et métapotamon). Sa présence marque l'apport, dans les eaux profondes des laurons, de formes potamobiontes depuis les grands canaux bordant les marais où on les a aussi récoltées.

\section{ChIronomides}

L'analyse du peuplement a été réalisée sur les populations larvaires et sur les populations imaginales.

Les premières nous ont permis de définir la structure globale des communautés aquatiques de Chironomides. En raison des difficultés de la systématique larvaire, le matériel récolté a été déterminé au niveau des sous-familles dont la valeur d'indicateurs écologiques est bien reconnue.

Les imagos ont été obtenus par élevages et par récoltes au filet entomologique; ils ont tous été déterminés jusqu'à l'espèce et nous ont ainsi fourni l'inventaire spécifique du peuplement chironomidien.

La communauté larvaire atteint sa densité maximum dans les stations à submersion semi-permanente $\left(\mathbf{S}_{2}\right.$ et $\left.\mathbf{S}_{8}\right)$. En fait la station $\mathbf{S}_{8}$, la plus riche en individus (2 100 individus récoltés sur un cycle annuel), est la plus pauvre en espèces ( 7 , seulement) ; ici, une seule espèce, Camptochironomus palidivittatus, constitue l'essentiel de l'effectif de ces Diptères. Par contre, dans les laurons $\left(S_{5}\right.$ et $\left.S_{6}\right)$, l'effectif de la population larvaire est de l'ordre de 200 individus pour l'année, mais avec une diversité spécifique élevée (20 espèces à $S_{5}, 17$ à $S_{6}$ ).

Sur un transect passant par les principales stations de la zone du Coucou, l'évolution de l'abondance relative des trois sous-familles (Orthocladiinae, Chironominae, Tanypodinae) apporte plusieurs informations (fig. 12) :

1. Les Chironominae dominent en effectif dans toutes les stations, sauf dans les laurons ouverts $\left(S_{5}\right)$; dans les stations $S_{2}$ et $S_{8}$ ils forment plus de $80 \%$ de l'effectif de la population larvaire.

La dominance des Chironominae est toujours liée à la présence d'une grande quantité de matière organique sur le substrat. Celui-ci est le siège d'oxydo-réductions; lorsque les réactions réductrices s'installent, les larves de Chironominae prolifèrent car elles sont alors les plus aptes à supporter les déficits en oxygène : "Une très 
grande abondance de Chironominae révèle des milieux pauvres en oxygène » (Tourenq 1975). Ainsi, à $S_{2}$ et $S_{8}$, la prolifération des Chironominae s'accompagne d'une complète disparition des Orthocladiinae.

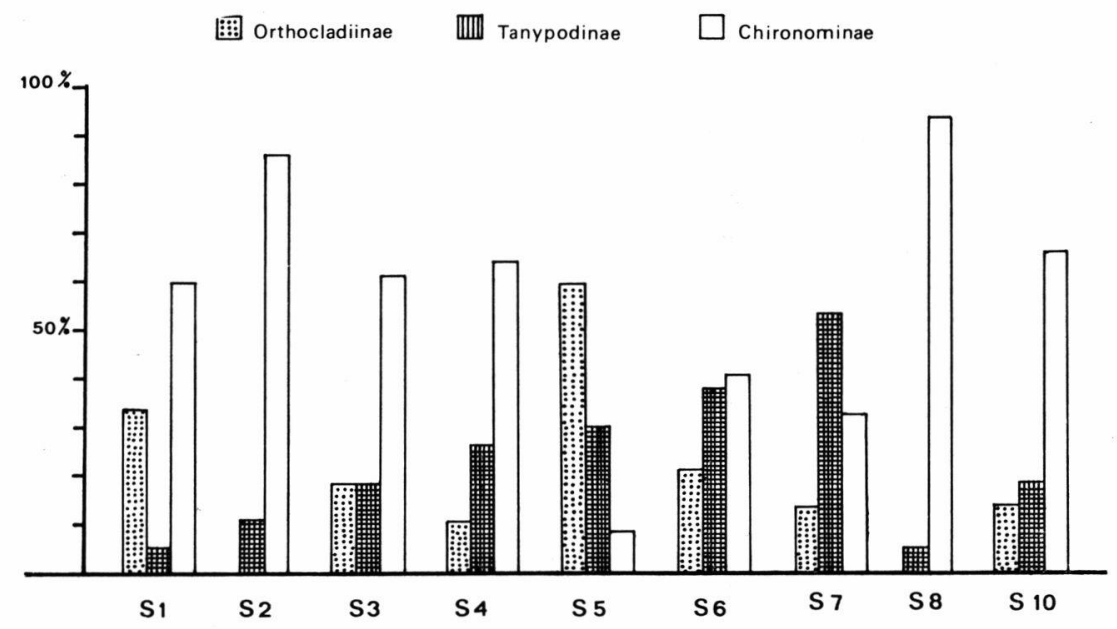

Fig. 12. - Structure de la communauté larvaire des Chironomides dans les différentes stations des marais du Coucou en fonction de l'abondance des trois sous-familles.

2. Un équilibre quantitatif entre les trois sous-familles s'établit lorsque, dans le biotope, sont associés un fond riche en matière organique et une eau renouvelée, comme c'est le cas dans les laurons colmatés $\left(\mathrm{S}_{6}\right)$.

3. Dans les laurons ouverts, les Orthocladiinae deviennent le groupe dominant.

Une telle organisation biotique du peuplement chironomidien ressemble à celles rencontrées dans les ruisseaux de haute montagne par Laville et Lavandier (1977), dans les milieux stagnants d'altitude par Laville (1972 )et dans le rhithron et l'épirhithron des grandes rivières comme le Lot par Laville (1979).

Elle semble confirmer l'hypothèse des botanistes Molinier et Tallon (1949) qui fait des laurons des zones refuges pour les espèces boréoalpines. On sait, en effet, que les larves d'Orthocladiinae sont primitivement adaptées aux milieux des régions froides (Oliver 1971). Cette famille domine dans les milieux des régions arctiques (Oliver 1968), diminue (Freeman 1955, 1956), ou disparaît (Fittkau 1964) dans les régions chaudes.

La plupart des 54 espèces de Chironomides inventoriées dans l'ensemble des marais du Coucou sont connues en Camargue (cf. Tourenq 
1975). Ce sont principalement des espèces eurythermes et saprophiles, inféodées aux biotopes lentiques.

Sur les 18 espèces qui ne figurent pas dans la faune de la Camargue, 11 sont cantonnées dans les laurons et leurs abords immédiats.

Tanypodinae :

Arctopelopia barbitarsis (Zett.)

Arctopelopia griseipennis (v.d. W.)

Orthocladiinae :

Corynoneura scutellata Winn.

Cricotopus lygropis Edw.

Gymnometriocnemus brumalis Edw.

Orthocladius rubicundus (Mg.)

Psectrocladius calcaratus Edw.

Psectrocladius conjugens (Br.)

Pseudorthocladius curtistylus (G.)

Chironominae :

Parachironomus varus $\mathrm{G}$.

Xenochironomus xenolabis $\mathbf{K}$.

Leur signification écologique mérite d'être analysée en tenant compte des particularités de leur distribution géographique et de leur habitat.

On doit noter avant tout la diversité des Orthocladiinae.

Parmi ces caractéristiques, deux espèces, seulement, ont déjà été signalées en Europe méditerranéenne :

- Parachironomus varus, qui a une vaste répartition européenne, jusque dans la haute plaine boréale (Fittkau et al. 1978).

- Xenochironomus xenolabis, qui a la même répartition européenne que $P$. varus mais est présente aussi en Afrique (Soudan, Ouganda) (Freeman 1957).

Les deux Arctopelopia sont connues du massif alpin, des massifs hercyniens occidentaux, de la grande plaine d'Europe centrale, des illes britanniques et de tout le bouclier finno-scandinave (Fittkau et al. 1978) : il s'agit d'espèces boréo-alpines sténothermes (Fittkau 1962).

Les Orthocladiinae : $C$. scutellata, G. brumalis, $O$. rubicundus, $P$. calcaratus et $P$. curtistylus présentent la même répartition biogéographique que les Arctopelopia. Elles se placent donc dans l'élément boréoalpin qui, de ce fait, domine parmi les Chironomides caractéristiques des laurons.

Deux espèces ont un aréal plus limité :

- Cricotopus lygropis connue des îles britanniques et, en France, de la région grenobloise (Serra-Tosio 1977). 
- Psectrocladius conjugens, connue de la grande plaine d'Europe centrale (Fittkau et al. 1978).

D'après leur distribution géographique et leur habitat, les sept espèces boréoalpines sont des formes d'eaux froides: C. Lygropis présente des exigences thermiques similaires. Les trois autres espèces sont plus eurythermes.

Les éléments faunistiques les plus originaux dans l'ensemble du peuplement aquatique de la zone du Coucou et, en même temps, les plus significatifs de la communauté animale des laurons, sont les espèces sténothermes et qui ont pour habitat normal les eaux courantes.

Dans cette catégorie nous retiendrons cinq espèces :

- Gymnometriocnemus brumalis et Orthocladius rubicundus vivent dans le crénon et le rhithron des massifs montagneux; elles ont été signalées dans l'Estaragne, torrent pyrénéen, vers 2000 mètres d'altitude (Laville et Lavandier 1977) (Lavandier 1979).

- Pseudorthocladius curtistylus vit dans le crénon, le rhithron et les eaux stagnantes (Fittkau et al. 1978).

- Cricotopus lygropis est cantonné dans l'épipotamon (Serra-Tosio 1977).

- Corynoneura scutellata vit dans le potamon et les eaux stagnantes (Fittkau et al. 1978); dans les Pyrénées, Laville (1972) l'a trouvée dans les eaux oligotrophes froides de lacs d'altitude.

Psectrotanypus varius vit uniquement dans les laurons ouverts. Tourenq (1975) l'a récoltée en Camargue dans les fossés de drainage abrités par la végétation où les amplitudes thermiques sont peu importantes.

\begin{tabular}{|c|c|c|c|c|c|}
\hline Biotopes & I & II & III & IV & $\mathrm{V}$ \\
\hline Arctopelopia barbitarsis $\ldots \ldots \ldots \ldots \ldots \ldots \ldots \ldots$ & + & & & & + \\
\hline Arctopelopia griseipennis $\ldots \ldots \ldots \ldots \ldots \ldots \ldots \ldots$ & + & & + & & + \\
\hline Psectrotanypus varius & & & & + & + \\
\hline Corynoneura scutellata & + & & & & + \\
\hline Cricotopus lygropis ............ & & & + & + & \\
\hline Gymnometrocnemus brumalis ........... & & + & + & & \\
\hline Orthocladius rubicundus ........... & & + & + & & \\
\hline Psectrocladius calcaratus $\ldots \ldots \ldots \ldots \ldots \ldots \ldots \ldots$ & & & & & + \\
\hline Psectrocladius conjugens $\ldots . \ldots \ldots \ldots$ & & & & & + \\
\hline p'seudorthocladius curtistylus $\ldots \ldots \ldots \ldots \ldots \ldots \ldots$ & + & + & + & & \\
\hline 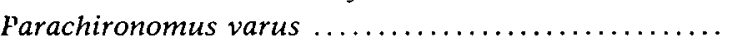 & & & & & + \\
\hline Xenochironomus xenolabis $\ldots \ldots \ldots \ldots \ldots \ldots \ldots$ & & & & + & + \\
\hline
\end{tabular}

Tableau I. - Répartition écologique des Chironomides caractéristiques des laurons. I : lacs d'altitude; II : crénon ; III : rhithron; IV : potamon; V : eaux stagnantes. 
Cette espèce a une très vaste distribution. Elle peut vivre dans le potamon et les eaux stagnantes; elle colonise aussi les sources limnohélocrènes du Holstein et est considérée en Europe comme sténotope (Fittkau 1962: 139̈).

Le tableau I résume la répartition écologique des Chironomides caractéristiques des laurons.

\section{5. - CONCLUSION : ORIGINALITE ECOLOGIQUE DES LAURONS}

De nombreuses données abiotiques, faunistiques et biogéographiques ont montré l'originalité du système hydrobiologique que constituent les laurons.

Nous avons recensé 28 espèces qui, dans le complexe de biotopes aquatiques que constituent les marais du Coucou, sont caractéristiques des laurons et de leur lisière. Elles présentent, pour la plupart, des exigences thermiques et des particularités d'habitat qui sont exceptionnelles dans un écosystème palustre soumis aux conditions du climat méditerranéen.

1) La présence de certaines espèces, dans les laurons et leur lisière, est déterminée par la température de l'eau. Elles sont connues, dans les autres régions de leur aire géographique, comme des formes sténothermes d'eau froide. Ces espèces ont aussi une distribution boréoalpine, les laurons de Crau constituant leur station la plus méridionale.

Dans cette catégorie, les espèces les plus représentatives sont l'Hydracarien Atractides prosiliens, le Cladocère Alona affinis et les Chironomides boréo-alpins.

L'hydroclimat créé dans les laurons par les résurgences de la nappe de Crau confère à ce biotope un caractère d'enclave froide de type septentrional, qui échappe largement aux caractéristiques bioclimatiques de la région méditerranéenne.

2) On relève aussi dans la communauté des laurons des espèces qui ont pour habitat les eaux courantes; ailleurs, elles vivent dans les ruisseaux de montagne (rhithron), les rivières (potamon) et, certaines, méme dans les sources.

La présence d'espèces du crénon revêt ici un intérêt particulier. En effet, dans les laurons, l'existence d'une eau froide renouvelée, d'origine souterraine, reproduit les conditions typiques d'une source limnocrène.

Un petit lot d'espèces révèle cette composante "crénale " de l'écosystème des laurons. Ce sont des formes crénobiontes, comme Pseudamnicola anatina et $P$ s. lanceolata, Atractides prosiliens (espèce 
vivant aussi dans les eaux souterraines), et des formes crénophiles comme Gymnometriocnemus brumalis, Orthocladius rubicundus, Pseudorthocladius curtistylus, ces dernières vivant aussi dans le rhithron.

Cependant, les espèces d'eau courante récoltées dans les laurons sont, en majorité, plutôt inféodées au potamon. C'est le cas de l'Oligochète Potamothrix heuscheri, du Décapode Atyaephyra desmarestii, des Chironomides Cricotopus lygropis, Corynoneura scutellata, Psectrotanypus varius.

Les Trichoptères des laurons sont à ranger aussi dans cette catégorie. Ainsi, Athripsodes dissimilis, dans le Doubs, est " typologiquement significative " du niveau 8 = épipotamon (Verneaux 1973); elle occupe la même zone écologique dans une rivière du Var, l'Argens (Dia 1977). Oxyethira flavicornis, Agrayela sexmaculata, Tricholeiochiton fagesi vivent dans les rivières à courant lent et dans les eaux stagnantes.

La présence d'espèces potamobiontes et surtout la dominance d'espèces d'eau calme dans le peuplement animal montre que les laurons s'intègrent dans l'écosystème lentique.

Cependant, le peuplement apparaît composite dans ses origines et traduit, en définitive, une mosaïque d'influences: lentique, crénale, rhithrale, potamale et même hypogée.

Néanmoins, la particularité remarquable de cette communauté est la présence d'espèces à la fois sténothermes froides et boréo-alpines. En cela les laurons apparaissent comme des refuges où ont pu survivre et se maintenir des espèces animales et végétales dont l'aréal s'étendait, aux périodes glaciaires, jusqu'à la région méditerranéenne.

\section{TRAVAUX CITES}

Aguesse (P.). 1961. - Contribution à l'étude écologique des Zygoptères de Camargue. Thèse doctorat d'Etat, Paris : 1-156.

Aguesse (P.). 1968. - Les Odonates de l'Europe occidentale, du nord de l'Afrique et des îles atlantiques. Masson \& $\mathrm{C}^{\mathrm{ie}}$. Paris.

Botosaneanu (L.). 1980. - Six nouvelles espèces ou sous-espèces de Trichoptères d'Europe méridionale (Trichoptera). Bull. zool. Mus. Univ. Amsterdam, 7 (17) : 165-179.

Dis (A.). 1977. - Etude écologique et essai de zonation d'une rivière côtière méditerranéenne. L'Argens (Var). Thèse $3^{e}$ cycle, Marseille : 1-155.

FirtKau (E. J.). 1962. - Die Tanypodinae (Dipt. Chironomidae). Abh. larvalsyst. Insekten, $6: 1-453$.

JirtKaU (E. J.). 1964. - Remarks on limnology of Central Amazon rainforest streams. Verh. Int. Verein. Limnol., 15 : 1092-1096.

FittKau (E. J.) et Reiss (F.). 1978. - in Limnofauna Europaea. Chironomidae Gustav Fischer Verlag. Stuttgart : 404-440.

Freeman (P.). 1955. - A study of African Chironomidae (Part I). Bull. Br. Mus. nat. Hist. B : 4 (1) : 1-67. 
Freeman (P.). 1957. - A study of the Chironomidae (Diptera) of Africa South of Sahara (Part II). Bull. Br. Mus. nat. Hist. B : 4 (7) : 286-368.

Freeman (P.). 1957. - Astudy of the Chironomidae (Diptera) of Africa South of the Sahara (Part III). Bull. Br. Mus. nat. Hist. B : 5 (9) : 324-426.

Giudicelli (J.) et Vaillant (F.). 1967. - La larve et la nymphe d'Allotrichia pallicornis Eaton (Trichoptera). Trav. Lab. Hydrobiol. Grenoble, 57-58 : 29-36.

LaNG. 1967. - Influence des rejets de la station d'épuration de Vidy sur la faune benthique du Leman. Verh. Internat. Verein Limnol., 19:1182-1192.

LAVANDiER (P.). 1979. - Ecologie d'un torrent pyrénéen en haute montagne : l'Estaragne. Thèse doctorat $\mathrm{d}^{\prime}$ Etat. Toulouse : 1-532.

Laville (H.). 1972. - Recherches sur les Chironomides (Diptères) lacustres du Massif du Néouvielle (Hautes-Pyrénées). Annls Limnol., 7 (2) : 173-332.

Laville (H.). 1979. - Etude de la dérive des exuvies nymphales des Chironomides au niveau du confluent Lot-Truyère. Annls Limnol., 15 (2) : 155-180.

I.Aville (H.) et LavandiER (P.). 1977). - Les Chironomides (Diptera) d'un torrent de haute montagne: l'Estaragne. Annls Liminol., 13 (1) : 57-81.

Marazanof (F.). 1969. - Contribution à l'étude des Mollusques des eaux douces et saumâtres de Camarque. I. Milieux et espèces. Annls Limnol., 5 (3) : 201323.

Metge (G.). 1977. - Etude synécologique de la dépression du Viguierat (B.-duRh.). Essai d'écologie quantitative en milieu hydromorphe et halophile. Thèse doctorat Université. Marseille 1-464.

Molinier (R.) et Tallon (G.). 1949. - La végétation de la Crau (Basse Provence). Rev. gen. Bot., $56: 1-130$.

Motas (C.). 1929. - Contribution à la connaissance des Hydracariens français particulièrement du Sud-Est de la France. Trav. Lab. Hydrobiol. Grenoble, 20 : 49-164.

Moubayed (Z.). 1978. - Etude écologique des marais du Sud de la Crau (Bouchesdu-Rhône). Analyse des peuplements d'invertébrés dulçaquicoles et de leurs relations avec 'hydroogie, la végétation et les influences humaines. Thèse $3^{\circ}$ cycle, Marseille : $222 \mathrm{p}$.

Nielsen (A.). 1948. - Postembryonic development and biology of the Hydroptilidae. K. danske vidensk. Selsk. Copenhagen, Biol. skr., S.l.

Oliver (D. R.). 1968. - Adaptations of Arctic Chironomidae. Ann.. Zool. Fenn. 5 : 111-118.

Oliver (D. R.). 1971. - Life history of the Chironomidae. A Rev. Ent., 16: 211225.

Pacaud (A.). 1939. - Ecologie des Cladocères. Bull. biol. France-Belgique, suppl. $25: 1-267$.

REY (J.). 1968. - Ecologie des Cladocères du Massif de Néouvielle (Haute-Pyrénées). Annls Limnol. 4 (3) : 325-356.

SARKKä (J.). 1978. - New records of profundal Oligochaeta from Finnish lakes with ecological observations. Ann. zool. Fennici $15: 235-240$.

Serra-Tosio (B.). 1977. - Notes sur les Diptères Chironomides de quelquse rivières polluées dans la région de Grenoble. Trav. Lab. Hydrobiol. Grenoble, $55: 83-88$.

TImm (T.). 1970. - On the fauna of the Estonian Oligochaeta. Pedobiologia, 10 : $52-78$.

Toureno (J.-N.). 1975. - Recherches écologiques sur les Chironomides (Diptera) de Camargue. Thèse doctorat d'Etat, Toulouse : 1-424.

Verneaux (J.). 1973. - Recherches écologiques sur le réseau hydrographique du Doubs. Essai de bioypologie. Ann. sci. Univ. Besançon, 9: 1-260.

VIETS (K. O.). 1978. in Limnofauna Europaea. Hydracarina. Gustav Fischer Verlag. Stuttgart : 154-181.

Willmann (R.) et Pieper (H.). 1978. - in Limnofauna Europaea. Lamellibranchiata. Gustav Fischer Verlag. Stuttgart : 135-137. 